Research, Society and Development, v. 9, n. 9, e598997642, 2020

(CC BY 4.0) | ISSN 2525-3409 | DOI: http://dx.doi.org/10.33448/rsd-v9i9.7642

Perfil de pacientes com criptococose em hospital regional do interior de São Paulo Profile of patients with cryptococcosis in a regional hospital in the interior of São Paulo Perfil de pacientes con criptococosis en un hospital regional en el interior de São Paulo

Recebido: 16/08/2020 | Revisado: 24/08/2020 | Aceito: 28/08/2020 | Publicado: 30/08/2020

Maikiane Aparecida Nascimento

ORCID: https://orcid.org/0000-0002-6843-2809

Hospital Estadual de Doenças Tropicais Dr. Anuar Auad/ Laboratório Estadual de Saúde Pública Dr. Giovanni Cysneiros, Brasil

E-mail: maikiane.nascimento@hotmail.com

Elorraine Coutinho Mathias Santos ORCID: https://orcid.org/0000-0003-4491-5635 Universidade do Oeste Paulista, Brasil E-mail: lohcoutinho_02@hotmail.com Valéria Moura de Carvalho

ORCID: https://orcid.org/0000-0002-5527-7643

Hospital Estadual de Doenças Tropicais Dr. Anuar Auad/ Laboratório Estadual de Saúde Pública Dr. Giovanni Cysneiros, Brasil E-mail: valeriamouracarvalho@hotmail.com

Marielly Sousa Borges

ORCID: https://orcid.org/0000-0003-0928-9771

Hospital Estadual de Doenças Tropicais Dr. Anuar Auad/ Laboratório Estadual de Saúde Pública Dr. Giovanni Cysneiros, Brasil E-mail: marielly.s.borges@ hotmail.com

João Pedro Brambilla Ederli ORCID: https://orcid.org/0000-0001-6254-9873 Universidade do Oeste Paulista, Brasil E-mail: jpbrambilla@outlook.com.br Camila Aparecida Nunes de Albuquerque ORCID: https://orcid.org/0000-0001-5241-8781 Hospital Estadual de Doenças Tropicais Dr. Anuar Auad/ Laboratório Estadual de Saúde Pública Dr. Giovanni Cysneiros, Brasil E-mail: camillaalbuquerque99@gmail.com 


\section{Savyla Franciele Soares Silva}

ORCID: https://orcid.org/0000-0002-5584-381X Hospital Estadual de Doenças Tropicais Dr. Anuar Auad/ Laboratório Estadual de Saúde Pública Dr. Giovanni Cysneiros, Brasil E-mail: savyla_ss1@hotmail.com Rosana Brambilla Ederli ORCID: https://orcid.org/0000-0003-2630-2464 Universidade Estadual Paulista, Brasil

E-mail: roederli@hotmail.com

Bruno Alexandre Soto Soto ORCID: https://orcid.org/0000-0002-9846-1918 Universidade do Oeste Paulista, Brasil E-mail: brunosoto@unoeste.br Alexandre Martins Portelinha Filho ORCID: https://orcid.org/0000-0003-1971-9923 Universidade do Oeste Paulista, Brasil E-mail: amportelinha@gmail.com

Daniela Vanessa Moris ORCID: https://orcid.org/0000-0002-0267-3589 Universidade do Oeste Paulista, Brasil E-mail: danimoris@gmail.com

\section{Resumo}

As infecções causadas por Cryptococus spp. são oportunistas, urbanas e associadas a imunossupressão. A mortalidade é estimada em $10 \%$ nos países desenvolvidos e $43 \%$ nos emergentes. O Objetivo foi avaliar os aspectos clínico-epidemiológicos da criptococose na região do oeste paulista. Trata-se de estudo transversal, baseado nas informações dos prontuários de pacientes em um hospital do interior de São Paulo, de 2006 a 2018. Aplicou-se análise descritiva por meio da frequência absoluta (n) e relativa (\%); e estatística através dos testes x2 e Exato de Fisher. Também foi calculado Risco Relativo (RR) e Odds Ratio (OD) para óbito. A neurocriptococose foi a principal manifestação clínica em 64,50\%. A pesquisa direta no líquor foi positiva em $85,71 \%$ das amostras, o imunodiagnóstico em 93,80\% e a cultura, padrão-ouro para fungo, em $55,55 \%$ dos casos. O teste $x^{2}$ e o Exato de Fisher não demonstraram diferença entre sexo, faixa etária, forma clínica ou óbito. RR e OD foram 
considerados baixos. A associação dos aspectos epidemiológicos da criptococose com seus sinais clínicos permite o diagnóstico precoce da doença, diminuindo o desenvolvimento de quadros graves. Além disso, as terapias antirretrovirais de alto desempenho diminuíram drasticamente a ocorrência de óbitos em pacientes com HIV.

Palavras-chave: Criptococose; HIV; Óbitos; Risco relativo; Odds Ratio.

\begin{abstract}
Infections caused by Cryptococus spp. are opportunistic, urban, and associated with immunosuppression. Mortality is estimated at $10 \%$ in developed countries and $43 \%$ in emerging countries. The objective was to evaluate the clinical-epidemiological aspects of cryptococcosis in the region of western São Paulo. This is a cross-sectional study, based on information from the medical records of patients in a hospital in the interior of São Paulo from 2006 to 2018. Descriptive analysis was applied through absolute (n) and relative (\%) frequency; and statistics through the x2 and Fisher's Exact tests. Relative Risk (RR) and Odds Ratio (OD) for death were also calculated. Neurocriptococcosis was the primary clinical manifestation of $64.50 \%$. The direct search for CSF was positive in $85.71 \%$ of the samples, the immunodiagnosis in $93.80 \%$ and the culture, gold standard for fungus, in $55.55 \%$ of the cases. The $\mathrm{x} 2$ test and Fisher's exact test showed no difference between sex, age group, clinical form of death. RR and OD were considered low. The association of the epidemiological aspects of cryptococcosis with its clinical signs allows the early diagnosis of the disease, reducing severe conditions. Besides, high-performance antiretroviral therapies have dramatically decreased the occurrence of deaths in patients with HIV.
\end{abstract}

Keywords: Cryptococcosis; HIV; Deaths; Relative risk; Odds ratio.

\title{
Resumen
}

Las infecciones causadas por Cryptococus spp. son oportunistas, urbanos y asociados con inmunosupresión. La mortalidad se estima en $10 \%$ en los países desarrollados y $43 \%$ en los países emergentes. El objetivo fue evaluar los aspectos clínico-epidemiológicos de la criptococosis en la región occidental de São Paulo. Este es un estudio transversal, basado en información de los registros médicos de pacientes en un hospital del interior de São Paulo, de 2006 a 2018. El análisis descriptivo se aplicó a través de la frecuencia absoluta (n) y relativa (\%); y estadísticas a través de las pruebas x2 y Fisher's Exact. También se calcularon el riesgo relativo (RR) y el odds ratio (OD) para la muerte. La neurocriptococosis fue la principal manifestación clínica en el $64.50 \%$. La búsqueda directa de LCR fue positiva en el 85,71\% de 
las muestras, el inmunodiagnóstico en el 93,80\% y el cultivo, patrón oro para hongos, en el $55,55 \%$ de los casos. La prueba $\mathrm{x} 2$ y la prueba exacta de Fisher no mostraron diferencias entresexo, grupo de edad, forma clínica o muerte. RR y OD se consideraron bajos. La asociación de los aspectos epidemiológicos de la criptococosis con sus signos clínicos permite el diagnóstico precoz de la enfermedad, reduciendo el desarrollo de afecciones graves. Además, las terapias antirretrovirales de alto rendimiento han disminuido drásticamente la aparición de muertes en pacientes con VIH.

Palabras clave: Criptococosis; VIH; Defunciones; Riesgo relativo; Odds ratio.

\section{Introdução}

O gênero Cryptococcus spp. abrange fungos da classe dos basidiomicetos que podem se transformar em leveduras. As espécies patogênicas são envolvidas por espessa cápsula polissacarídica, sendo esta a principal característica morfológica deste grupo e importante fator de virulência (Zaragoza, 2019). Apesar de existirem mais de 50 espécies do gênero, as duas principais responsáveis pela grande maioria das infecções em humanos são Cryptococcus neoformans e Cryptococcus gattii (Maziarz \& Perfect, 2016).

Até a década de 80 esta doença recebeu pouca notoriedade, porém com o crescimento do número de pacientes com o vírus da imunodeficiência humana (HIV) notou-se que mais de $80 \%$ das infecções criptocócicas na época foram relatadas nesse grupo de pacientes, com uma prevalência variando de 2 a 10\% nos Estados Unidos, Europa Ocidental e Austrália e 5 a 30\% na África (Colombo, et al., 2015). A mortalidade geral é estimada em $10 \%$ nos países desenvolvidos e 43\% nos países emergentes (Hurtado, et al., 2019). A mortalidade foi estimada em $70 \%$ em países de baixa renda, $40 \%$ em países de renda média, 20 a 30\% na Europa e $20 \%$ na América do Norte (Mourad \& John, 2018).

As infecções causadas pelo complexo de espécies C. neoformans são oportunistas, urbanas e geralmente associadas a condições de imunossupressão (Andrade-Silva, et al., 2018). Já a espécie C. gattii, em particular, é reconhecida como causadora de meningite em pessoas imunocompetentes em pacientes de regiões endêmicas da África, Índia, América do Sul, Norte da Austrália e Papua Nova Guiné (Acheson, et al., 2018).

Nos imunossuprimidos manifesta-se geralmente como meningite ou meningoencefalite aguda, subaguda ou crônica, caracterizada por cefaleia, febre, alteração do nível de consciência, confusão mental, perda da memória, perda da visão, convulsão, coma, fotofobia e rigidez na nuca. As principais complicações encontradas são hipertensão 
intracraniana, hidrocefalia e, menos frequentemente, a paralisia dos pares cranianos (Hurtado, et al., 2019).

O diagnóstico laboratorial é constituído principalmente de detecção direta do agente em fluido biológico, pesquisa do antígeno capsular criptocócico, cultivo e exame anatomopatológico. A coleta do líquor é a principal ferramenta no diagnóstico precoce da infecção no sistema nervoso central. Em pessoas vivendo com HIV/Aids (PVHA) com sintomas neurológicos, a coleta de líquor deve ser realizada o mais precoce possível (WHO, 2018).

O tratamento antifúngico depende da forma clínica da criptococose e do estado imunológico do paciente. $\mathrm{O}$ atual arsenal antifúngico disponível para o tratamento da criptococose é limitado a três drogas, usadas isoladamente ou em combinação: Desoxicolato de anfotericina B e suas formulações lipídicas e dispersão coloidal, flucitosina e fluconazol (Spadari, et al., 2020). O consenso recomenda o uso de anfotericina desoxicolato e 5fluocitosina durante pelo menos duas semanas, seguido de fluconazol por pelo menos oito semanas (Skipper, et al., 2019). Pela grande importância da criptococose como problema de saúde pública, o presente trabalho objetivou determinar a magnitude da infecção em um hospital de urgência do interior de São Paulo.

\section{Metodologia}

Trata-se de um estudo transversal, quantitativo, baseado em dados provenientes dos prontuários dos pacientes atendidos em um hospital regional do interior de São Paulo, no período de 2006 a 2018. As variáveis analisadas foram idade, sexo, forma clínica, ano de diagnóstico, comorbidades e exames laboratoriais. Foram feitas tabelas de distribuição de frequências absolutas e relativas. As variáveis também foram analisadas por meio dos testes x2 e do Exato de Fisher no software Microsoft Excel®. Foi considerado significativo o valor de p<0,05. Foi determinado o Risco Relativo (RR), a Razão de Prevalência e Odds Ratio (OD). O Risco Relativo e o Odds Ratio são considerados relevantes com valores >1 (Szklo, 2015). Este trabalho foi aprovado pelo Comitê de Ética em Pesquisa da instituição proponente com parecer número CAAE - 58663916.6.0000.5515. 


\section{Resultados e Discussão}

Foram identificados 61 casos da doença, dos quais apenas 31 prontuários estavam disponíveis para coleta de informações. Dos prontuários analisados houve um predomínio de pacientes do sexo masculino em relação ao feminino na proporção de 3:1. A média de idade foi de 41,50 anos com desvio padrão de 16,16 anos. As idades mínima e máxima foram respectivamente 21 e 81 anos. Os anos de maiores incidências foram 2007, 2008 e 2009. Já os anos de menores incidências foram 2014, 2016 e 2018. (Tabela 1).

Tabela 1 - Perfil clínico-epidemiológico de 31 pacientes diagnosticados com criptococose em hospital regional do interior de São Paulo, 2006-2008.

\begin{tabular}{|c|c|c|c|c|c|}
\hline & \multicolumn{4}{|c|}{ Frequência } & \multirow[t]{3}{*}{ Valor de $p$} \\
\hline & \multicolumn{2}{|c|}{ Masculino } & \multicolumn{2}{|c|}{ Feminino } & \\
\hline Variável & $\mathbf{N}$ & $\%$ & $\mathbf{N}$ & $\%$ & \\
\hline \multicolumn{6}{|l|}{$\operatorname{sexo}(n=31)$} \\
\hline & 23 & 74,19 & 8 & 25,81 & \\
\hline \multicolumn{6}{|l|}{ Faixa etária } \\
\hline $00-29$ & 7 & 22,58 & 1 & 03,37 & \\
\hline $30-59$ & 13 & 41,93 & 4 & 12,90 & $0,268553854^{\mathrm{a}}$ \\
\hline$\geq 60$ & 3 & 09,61 & 3 & 09,61 & \\
\hline \multicolumn{6}{|l|}{ Formas clínicas } \\
\hline Meníngea & 11 & 35,48 & 4 & 12,90 & \\
\hline Pulmonar & 0 & 00,00 & 1 & 03,37 & \\
\hline Meníngea + pulmonar & 3 & 09,61 & 0 & 00,00 & $0,47490643^{\mathrm{a}}$ \\
\hline Disseminada & 1 & 03,37 & 0 & 00,00 & \\
\hline Outras formas & 4 & 12,90 & 1 & 03,37 & \\
\hline Não esclarecida & 4 & 12,90 & 2 & 06,10 & \\
\hline \multicolumn{6}{|l|}{ Comorbidades } \\
\hline Hepatite & 4 & 12,90 & 0 & 00,00 & \\
\hline HIV & 9 & 29,04 & 0 & 00,00 & \\
\hline Outras infeccões & 7 & 22,58 & 1 & 03.37 & \\
\hline Doenca autoimune & 0 & 00,00 & 3 & 09.61 & \\
\hline Distúrbios metabólicos* & 1 & 03,37 & 2 & 06,10 & \\
\hline Outras & 5 & 16,12 & 0 & 00,00 & \\
\hline Sem comorbidades & 6 & 19,35 & 4 & 12,90 & \\
\hline
\end{tabular}

\footnotetext{
*Hipertensão, diabetes, dislipidemias; a - Teste $x^{2}$. Fonte: Autores.
} 
Research, Society and Development, v. 9, n. 9, e598997642, 2020

(CC BY 4.0) | ISSN 2525-3409 | DOI: http://dx.doi.org/10.33448/rsd-v9i9.7642

A neurocriptococose foi a principal manifestação da doença em $64,50 \%$ dos casos, seguida das formas pulmonar, forma disseminada, forma não esclarecida e outras. O teste quiquadrado não demonstrou diferença entre gênero e a faixa etária [ $p=0,268553854]$ ou forma clínica $[p=0,47490643]$.

A confirmação do diagnóstico foi estabelecida a partir do exame direto do líquor in natura por intermédio de coloração pela tinta Nanquim, ou indiretamente, mediante presença do antígeno capsular para Cryptococcus spp. através da aglutinação em látex (Tabela 2).

Ocorreu um número total de seis óbitos (19,36\%). Pessoas vivendo com HIV/Aids corresponderam a nove do total de casos da doença $(29,03 \%)$, e destes apenas 1 foi a óbito, contra uma taxa de letalidade de $19 \%$ em pacientes sem o vírus $(n=5)$.

Os testes estatísticos não demonstraram diferença de óbito entre pacientes portadores do HIV comparado aos demais (Tabela 3). Nos pacientes com o vírus, a contagem de linfócitos T CD4+ esteve disponível em 44,44\% de casos, com valores que variavam de 6 a 162 células/ $\mu \mathrm{L}$ (média de 69 células/ $\mu \mathrm{L}$ ).

Tabela 2 - Metodologias empregadas no diagnóstico de 31 pacientes com criptococose em hospital regional do interior de São Paulo, 2006-2008.

\begin{tabular}{lllll}
\hline \multicolumn{5}{c}{ Frequência } \\
\hline & \multicolumn{2}{c}{ Positivo } & \multicolumn{2}{c}{ Negativo } \\
\hline Metodologias empregadas & N & \% & N & \% \\
\hline & & & & \\
\hline Pesquisa direta (n=14) & 12 & 85,71 & 2 & 14,29 \\
Látex (n=16) & 15 & 93,75 & 1 & 06,25 \\
Anatomopatológico (n=1) & 1 & 100 & 0 & 00,00 \\
Cultura (n=9) & 5 & 55,55 & 4 & 44,45 \\
\hline
\end{tabular}

Fonte: Autores.

Tabela 3 - Risco relativo, ODDS ratio e comparação de óbito entre pacientes com e sem HIV diagnosticados com criptococose em hospital regional do interior de São Paulo, 2006-2008.

\begin{tabular}{|c|c|c|c|c|c|c|c|}
\hline & Óbito & & Total & $\mathbf{R P}$ & $\mathbf{R R}$ & $O D$ & $p<0,001$ \\
\hline & Sim & & Não & \multirow{3}{*}{0,4889} & \multirow{3}{*}{0,4888} & \multirow{3}{*}{0,4250} & \multirow{3}{*}{$0,6423^{b}$} \\
\hline HIV+ & 1 & 9 & 8 & & & & \\
\hline HIV - & 5 & 22 & 17 & & & & \\
\hline Total & 6 & 31 & 25 & & & & \\
\hline
\end{tabular}

$\mathrm{b}-$ Teste Exato de Fisher. Fonte: Autores. 
A neurocriptococose é descrita como a forma clínica mais frequentemente observada, ocorrendo em mais de $80 \%$ dos pacientes, seja isolada ou em associação a forma pulmonar (Colombo, 2015). Em nosso trabalho observamos uma prevalência dessa forma clínica em $64,5 \%$ dos casos. O envolvimento pulmonar na criptococose é considerado o segundo mais frequente após o acometimento do sistema nervoso central, dado também observado neste estudo.

Dentre as micoses sistêmicas, a criptococose tem sido relatada como a mais prevalente em termos de internação. Dados do Sistema de Internação Hospitalar do Sistema Único de Saúde (SIH/SUS-DATASUS) demonstram que a criptococose apresentou o maior número de internações no período de 2007 a 2008 em todo o país, sendo este achado concordante com nossos resultados. Nas regiões Sul e Sudeste do Brasil predominam a criptococose associada à Aids em homens, causada pela variedade $C$. neoformans, e apresenta uma letalidade de cerca de 40\% (SUVISA/GO, 2012).

Segundo a literatura, devido ao seu caráter predominante de infecção oportunista, a criptococose é mais prevalente em humanos com condições de risco, principalmente com imunodepressão pelo HIV, linfomas, leucemias, uso de corticoides e transplantes (WHO, 2018). Neste espaço amostral, a maior parte dos casos (71\%) não ocorreu em pacientes com diagnóstico de HIV, discordando de autores que asseguram ser este o grupo mais acometido mundialmente pela criptococose, pois a falta de células de defesa predispõe esses pacientes ao desenvolvimento dessa patologia (Candiani, 2007).

Além disso, diferente do que tem sido descrito na literatura, observamos uma baixa letalidade em pacientes portadores de HIV, apenas um veio a falecer dos nove casos, correspondendo a uma taxa de letalidade de apenas 11,11\%. A discordância do presente ensaio pode estar relacionado à perda de dados, já que apenas 31 dos 66 prontuários estavam disponíveis para obtenção de dados, resultando em possível subestimação do número de pacientes portadores do HIV que foram a óbito. Ou talvez esteja associado ao fato de que as terapias antirretrovirais estão cada vez mais eficientes na manutenção da doença (Nunes, 2015).

Em detrimento da limitação do estudo, outros trabalhos têm demostrado diminuição da prevalência de óbito por diversas doenças oportunistas em PVHA (Nunes, et.al., 2015). Na análise multivariada de Candiani et al. (2007) foi observado que o risco relativo de desenvolvimento de infecção oportunista foi 3,3 vezes maior para hospitalizações, antes da terapia antirretroviral de alta eficácia (HAART); assim como, houve um declínio de 38\% nos óbitos com a incorporação desta nova terapia. 
A introdução da HAART contribuiu para mudança de perfil da doença para um quadro crônico tratável, diminuição nas taxas de óbito, redução significativa de doenças oportunista, e consequentemente, expressiva melhora da qualidade de vida. Contudo, ainda se questiona a eficácia desta terapia, especialmente em pacientes com baixa adesão ao tratamento (Candiani, et al., 2007).

Em relação ao diagnóstico, neste estudo o método mais utilizado para o diagnóstico laboratorial foi a pesquisa do antígeno polissacarídeo circulante no soro e líquor através da prova de látex. Essa prova é considerada uma técnica muito sensível e específica na detecção dessa micose, além de ser rápida, de fácil execução e baixo custo. Nesta investigação o antígeno capsular foi positivo em 93,0\% dos testes. Resultados falso-negativos podem acontecer em situações em que há excesso de antígeno, nomeadamente efeito pós-zona. Resultados falso-positivos ocorrem em pouquíssimas ocasiões, tais como, nos casos de reações cruzadas com outros microrganismos e com o fator reumatoide (Bastos, 2017).

A cultura considerada o exame comprobatório da doença foi realizada em apenas $16,12 \%$ das amostras $(n=9)$ e positivação em apenas 55,55\%. Atribui-se essa baixa sensibilidade ao fato de que a maioria das coletas ocorre após o início da terapia antifúngica, comprometendo o crescimento do fungo. Conforme o último Consenso Brasileiro de Criptococose, se coletada adequadamente a cultura apresenta sensibilidade de até $89 \%$ dos pacientes não infectados pelo HIV e em 95\% a 100\% dos pacientes com Aids (Brazilian Society of Tropical Medicine, 2008).

Para evidenciar a presença dessa levedura nos tecidos, bem como caracterizar a resposta inflamatória, pode-se também recorrer às técnicas anatomopatológicas. No presente trabalho, apenas um caso foi concluído após a realização deste exame. Essa metodologia permite analisar amostras dos mais variados tecidos e órgãos, sendo as leveduras encontradas principalmente em fragmentos de cérebro e pulmão. Colorações como a mucicarmim de Mayer permitem a visualização da cápsula que se cora fortemente de vermelho. Já a coloração de Fontana-Masson evidencia o depósito de melanina produzida por fungos desse gênero (Colombo, 2015).

Complementarmente a essas técnicas, as metodologias que empregam testes imonocromatográficos têm se destacado como ensaios rápidos, a preço acessível, de fácil execução, e que detectam precocemente diversas doenças. A OMS recomenda o ensaio de fluxo lateral de antígeno criptocócico (CrAg LFA) para detecção do patógeno em diversas amostras biológicas como o soro, líquor e até mesmo a urina (WHO, 2018). 
Com isso concluímos que o cenário de criptococose tem mudado drasticamente. O uso de antirretrovirais de alta eficiência em pacientes imunocomprometidos diminuíram significativamente a incidência e morbimortalidade por essa doença em pacientes com HIV.

\section{Considerações Finais}

A criptococose não é a doença fúngica de maior ocorrência em animais e no homem, porém o conhecimento de seus aspectos epidemiológicos, relacionados principalmente à transmissão, é importante para prevenir infecções. Além disso, a associação desses aspectos com os sinais clínicos permite o diagnóstico precoce da doença a fím de impedir o desenvolvimento de quadros graves, que podem levar os pacientes a óbito. Entre as limitações do estudo, relatamos a ausência de vários prontuários com casos confirmados no sistema eletrônico e o fato do pequeno tamanho da amostra, caracterizada como de conveniência; o que pode ter levado à ausência de associações.

Dentre as sugestões recomendamos o documento recentemente publicado pela Organização Mundial da Saúde (OMS), baseado em evidências, que sumariza as principais recomendações para o diagnóstico e tratamento da criptococose em pacientes infectados pelo HIV (WHO, 2018). Também salientamos a pertinência da realização de novos estudos sobre esse agravo, para maior análise situacional da doença não somente no estado de São Paulo, mas em todo país.

\section{Referências}

Acheson, E. S., Galanis, E., Bartlett, K., Mak, S., \& Klinkenberg, B. (2018). Searching for clues for eighteen years: Deciphering the ecological determinants of Cryptococcus gattii on Vancouver Island, British Columbia. Medical Mycology, 56(2), 129-144. doi:10.1093/mmy/myx037

Andrade-Silva, L. E., Ferreira-Paim, K., Ferreira, T. B., Vilas-Boas, A., Mora, D. J., Manzato, V. M., Fonseca, [..], \& Silva-Vergara, M. L. (2018). Genotypic analysis of clinical and environmental Cryptococcus neoformans isolates from Brazil reveals the presence of VNB isolates and a correlation with biological factors. PloS one, 13(3), 82 p. doi:10.1371/journal.pone.0193237 
Bastos A. L. (2017). Aspectos clínicos-epidemiológicos da criptococose no estado de Goiás. Dissertação de Mestrado em Ciências da Saúde, Universidade Federal de Goiás - UFG, Goiânia, Brasil.

Brazilian Society of Tropical Medicine. Guidelines in cryptococcosis-2008. (2008). Rev. Soc. Bras. Med. Trop, 41(5), 524-544. doi:10.1590/S0037-86822008000500022

Candiani, T. M. S., Pinto, J., Cardoso, C. A. A., Carvalho, I. R., Dias, A. C. M., Carneiro, M., \& Goulart, E. A. (2007). Impact of highly active antiretroviral therapy (HAART) on the incidence of opportunistic infections, hospitalizations and mortality among children and adolescents living with HIV/AIDS in Belo Horizonte, Minas Gerais State, Brazil. Cadernos de Saúde Pública, 23 (Suppl. 3), S414-S423. doi:10.1590/S0102-311X2007001500009

Colombo, G., Táparo, C. V., Araújo Júnior, E. C., Makatu, M. Y., Santos, F. S., \& Marinho, M. (2015). Caracterização bioquímica e molecular de Cryptococcus spp. isolados de excretas ambientais de pombos (Columba livia domestica). Arquivo Brasileiro de Medicina Veterinária e Zootecnia, 67(6), 1639-1645. doi:10.1590/1678-4162-7753

Hurtado, J. C., Castillo, P., Fernandes, F., Navarro, M., Lovane, L., [..], \& Casas, I. (2019). Mortality due to Cryptococcus neoformans and Cryptococcus gattii in low-income settings: an autopsy study. Scientific reports, 9(1): 1-10. doi:10.1038/s41598-019-43941-w

Maziarz, E. K., \& Perfect, J. R. (2016). Cryptococcosis. Infectious disease clinics of North America, 30(1), 179-206. doi:10.1016/j.idc.2015.10.006

Mourad, A., \& Perfect, J. R. (2018). Present and Future Therapy of Cryptococcus. Infections. Journal of fungi, 4(3), 79. doi:10.3390/jof4030079

Nunes, A. A., Caliani, L. S., Nunes, M. S., Silva, A. S., \& Mello, L. M. (2015). Análise do perfil de pacientes com HIV/Aids hospitalizados após introdução da terapia antirretroviral (HAART). Ciência \& Saúde Coletiva, 20(10), 3191-3198. doi:10.1590/1413812320152010.03062015 
Skipper, C., Abassi, M., \& Boulware, D. R. (2019). Diagnosis and Management of Central Nervous System Cryptococcal Infections in HIV-Infected Adults. Journal of fungi (Basel, Switzerland), 5(3), 65-76. doi:10.3390/jof5030065

Spadari, C. C, Wirth, F., Lopes, L. B, \& Ishida, K. (2020). Novas abordagens para o tratamento de criptococose. Microorganismos, 8 (4),

613. doi:10.3390/microorganisms8040613

Secretaria de Saúde do Estado de Goiás - SUVISA. (2012). Micoses Sistêmicas. Recuperado de https://www.saude.go.gov.br/sesg/comissao-de-integracao-ensino-servico/337suvisa/7428-micoses-sist.

WHO, World Health Organization. (2018). The diagnosis, prevention and management of cryptococcal disease in hiv-infected adults, adolescents and children. Recuperado de https://www.who.int/hiv/pub/guidelines/cryptococcal-disease/en/.

Zaragoza, O. (2019). Basic principles of the virulence of Cryptococcus. Virulence, 10(1), 490-501. doi:10.1080/21505594.2019.1614383

\section{Porcentagem de contribuição de cada autor no manuscrito}

Maikiane Aparecida Nascimento - 30\%

Elorraine Coutinho Mathias Santos - 05\%

Valéria Moura de Carvalho - 05\%

Marielly Sousa Borges $-05 \%$

João Pedro Brambilla Ederli - 05\%

Camila Aparecida Nunes de Albuquerque - 05\%

Savyla Franciele Soares Silva - 05\%

Rosana Brambilla Ederli - 05\%

Bruno Alexandre Soto - 05\%

Alexandre Martins Portelinha Filho - 05\%

Daniela Vanessa Moris - 25\% 